\title{
TIGHT PROJECTIONS OF FRAMES ON INFINITE DIMENSIONAL HILBERT SPACES
}

\author{
JOHN JASPER
}

Abstract. We characterize the frames on an infinite dimensional separable Hilbert space that can be projected to a tight frame for an infinite dimensional subspace. A result of Casazza and Leon states that an arbitrary frame for a $2 N-$ or $(2 N-1)$-dimensional Hilbert space can be projected to a tight frame for an $N$-dimensional subspace. Surprisingly, we demonstrate a large class of frames for infinite dimensional Hilbert spaces which cannot be projected to a tight frame for any infinite dimensional subspace.

Mathematics subject classification (2010): Primary: 46C05; Secondary: 47A20.

Keywords and phrases: Frames, projections of frames, compressions.

\section{REFERENCES}

[1] P. G. CasazZa, M. Leon, Projections of frames, Proc. SPIE 5914, 591402 (2005), 277-289.

[2] O. Christensen, An introduction to frames and Riesz bases, Appl. Numer. Harmon. Anal., Birkhäuser Boston, Inc., Boston, MA, 2003.

[3] J. B. Conway, A course in functional analysis, Second edition, Graduate Texts in Mathematics, Springer-Verlag, New York, 1990.

[4] M. S. Copenhaver, Y. H. Kim, C. Logan, K. Mayfield, S. K. Narayan, M. J. Petro, J. SHEPERD, Diagram vectors and tight frame scaling in finite dimensions, preprint, 2012.

[5] M. Fickus, D. G. MiXon AND M. J. POTEET, Frame completions for optimally robust reconstruction, preprint arXiv:1107.1912, 2011.

[6] G. Kutyniok, K. A. Okoudjou, F. Philipp, E. K. Tuley, Scalable frames, preprint arXiv:1204.1880v3, 2012.

[7] P. Massey, M. Ruiz, D. Stojanoff, Optimal completions of a frame, preprint arXiv:1206.3588v1, 2012. 